\title{
ON TEICHMÜLLER CONTRACTION
}

\author{
FREDERICK P. GARDINER
}

(Communicated by Clifford J. Earle, Jr.)

\begin{abstract}
Universal Teichmüller space is the space of quasi-symmetric homeomorphisms $Q S$ of a circle factored by those Möbius transformations that preserve the circle. Another Teichmüller space, which also has universal properties, is $Q S$ factored by the closed subgroup $S$ of symmetric homeomorphisms. Teichmüller's metric for $Q S$ mod $S$ is the boundary dilatation metric. Sullivan's coiling property for Beltrami lines and the Hamilton-Reich-Strebel necessary and sufficient condition for extremality are proved for $Q S \bmod S$. The coiling property implies a contraction principle for certain types of selfmappings of Teichmüller space. It is also shown that the boundary dilatation metric has an infinitesimal form and that this metric is the integral of its infinitesimal form.
\end{abstract}

\section{INTRODUCTION}

The principle of Teichmüller contraction concerns curves in Teichmüller space which are approximate geodesics with respect to Teichmüller's metric. More precisely, it concerns curves, which are of the special form $[t \mu],-1 \leq$ $t \leq 1$, for some Beltrami coefficient. We will call such curves Beltrami lines. It is known that such a curve is a geodesic if $\mu$ is extremal [7]. Moreover, for extremal $\mu$ the mapping $I_{\mu}$ from the open interval $-1<t<1$ with the Poincaré metric into Teichmüller's space with Teichmüller's metric given by $t \mapsto\left[t \mu /\|\mu\|_{\infty}\right]$ is an isometry.

Whether or not $\mu$ is extremal, the mapping $I_{\mu}$ is weakly contracting. Teichmüller contraction says that if the mapping $I_{\mu}(t)$ fails to preserve distance between two points then it is strictly contracting at all pairs of points on the same Beltrami line and within a specified distance from the two given points. Moreover, the amount by which it is contracting is independent of $\mu$. This property of the mapping $I_{\mu}$ is called a coiling property by Sullivan [11] because the Beltrami line $[t \mu]$ is similar to a flexible coiled wire; if two points along the line are not stretched apart as far as they can be by a certain factor, then throughout most of the line the distance between pairs of points is not as great as it could be, by an amount depending on the given factor.

Teichmüller contraction is closely related to a theorem of Royden [10], which asserts that for finite-dimensional Teichmüller spaces with complex structure,

Received by the editors November 14, 1991.

1991 Mathematics Subject Classification. Primary 30C60, 30C70, 32G15.

Partially supported by the National Science Foundation. 
Teichmüller's metric coincides with the Kobayashi metric. This theorem is extended to infinite-dimensional Teichmüller spaces with complex structure in [3]. A consequence of this theorem is that any holomorphic mapping between Teichmüller spaces and, in particular, from the unit disk with the Poncare metric into any Teichmüller space is weakly contracting.

Teichmüller contraction is more restrictive than Royden's theorem because it concerns only a special class of mappings from a disk or a line segment into a Teichmüller space, namely, those that are Beltrami lines, but it is more general because the constant of strict contraction is uniform over all $\mu$ and the emphasis is that the principle applies to infinite-dimensional spaces.

Teichmüller contraction applies in another setting, which is important in the study of dynamical systems. Consider the group $Q S$ of all quasi-symmetric homeomorphisms of a circle. Universal Teichmüller space is $Q S$ factored by the Mobius group $\operatorname{PSL}(2, \mathbb{R})$; but $Q S$ contains another topological subgroup, which is much larger than $\operatorname{PSL}(2, \mathbb{R})$, namely, the subgroup $S$ of symmetric homeomorphisms. $S$ can be defined as the closure in the quasi-symmetric topology of the group of real analytic homeomorphisms of the circle. $Q S \bmod S$ is another type of Teichmüller space with a complex structure and with a natural boundary dilation metric, which turns out to be the same as the quotient metric to Teichmüller's metric on $Q S \bmod \operatorname{PSL}(2, \mathbb{R})$ [5].

It is not known whether the Teichmüller metric on $Q S \bmod S$ coincides with the Kobayashi metric. Nonetheless, Teichmüller contraction applies in this setting and provides a method for showing that certain self-mappings of Teichmüller spaces are strict contractions, which therefore have unique fixed points. The goal is to apply this lemma to renormalization. This program is carried through by Sullivan in [11] for renormalization self-mappings of Teichmüller spaces of Riemann surface laminations.

This paper is divided into four sections. The first reviews the definitions of Poincaré's and Teichmüller's metrics and also the quotient Teichmüller metric on $Q S \bmod S$. In this section it is shown that the quotient metric on $Q S$ mod $S$ has an infinitesimal form, expressed in terms of degenerating sequences of quadratic differentials of norm 1, and it is shown that this quotient metric is the integral of its infinitesimal form. A key element in finding the infinitesimal form of Teichmüller's metric for $Q S$ mod $S$ is a theorem of Fehlmann [2] relating local dilatation of a quasi-symmetric mapping to boundary dilatation.

The second section gives a precise statement of Teichmüller contraction, which applies to universal Teichmüller space, to the Teichmüller space of a Fuchsian group, and to $Q S \bmod S$.

The third section connects the near extremality of a mapping to the near extremality of a linear functional, called the Hamilton functional. The proof of this connection is based on the main inequality of Reich and Strebel [9], which is a form of Grötzsch's inequality. The main result is that a mapping is $\varepsilon$-extremal if and only if the Hamilton functional is $\varepsilon^{\prime}$-extremal, where $\varepsilon$ and $\varepsilon^{\prime}$ converge simultaneously to zero. A version of this result for Beltrami coefficients with constant absolute value was proved also by Reich and Strebel. The case when $\varepsilon$ and $\varepsilon^{\prime}$ are both equal to zero includes the proof of the Hamilton-Reich-Strebel theorem. In the setting of $Q S$ mod $S$, the parallel result gives a new form of this theorem. 
The fourth section gives the proof of Teichmüller contraction.

A forthcoming paper develops a parallel theory for the Teichmüller space of a polynomial-like mapping acting on the exterior of a connected filled-in Julia set. The $Q S$ mod $S$ Teichmüller space gives a convenient setting for parametrizing the exterior classes of such a mapping. There, the holomorphic quadratic differentials become lacunary series.

\section{A REVIEW OF PoINCARÉ's AND TeICHMÜlleR's METRICS}

Let $\Delta$ be the open unit disk and $L_{\infty}(\Delta)$ the Banach space of bounded, measurable, complex-valued functions defined on $\Delta$ with the essential supremum norm. For any $\mu$ in $M$, the open unit ball of $L_{\infty}(\Delta)$, there exists a quasiconformal self-mapping $f$ of $\Delta$, which satisfies the Beltrami equation [1]

$$
f_{\bar{z}}(z)=\mu(z) f_{z}(z) \text {. }
$$

Any two solutions of (1) are equal up to postcomposition by a Möbius transformation preserving $\Delta$. Thus, if we normalize a solution to (1) at three boundary points of $\Delta$, a solution is uniquely determined by $\mu$ and we may write $f=f^{\mu}$.

The universal Teichmüller space $T$ can be viewed as $M$ factored by an equivalence relation. Let $f_{0}$ and $f_{1}$ be solutions of (1) normalized at the same three points with Beltrami coefficients $\mu_{0}$ and $\mu_{1}$. We say $\mu_{0}$ and $\mu_{1}$ are equivalent if $f_{0}$ and $f_{1}$ are identical on the boundary of $\Delta$.

For any Fuchsian group $\Gamma$ acting on $\Delta$ and any closed subset $C$ of the boundary of $\Delta$, which contains the limit set of $\Gamma$ and which is invariant under $\Gamma$, the Teichmüller space $T(\Gamma, C)$ can be defined analogously. The set $M(\Gamma)$ consists of those elements of $M$ that are invariant as $(-1,1)$-differentials for $\Gamma$. That is, $\mu$ is in $M(\Gamma)$ if $\mu$ is in $M$ and

$$
\mu(B(z)) \overline{B^{\prime}(z)} / B^{\prime}(z)=\mu(z)
$$

for every $B$ in $\Gamma$. The Teichmüller space $T(\Gamma, C)$ is $M(\Gamma)$ factored by an equivalence relation depending on $C$; two Beltrami coefficients $\mu_{0}$ and $\mu_{1}$ are equivalent if $f_{0}$ and $f_{1}$ are identical on $C$. We often suppress $\Gamma$ and $C$ in the notation $T(\Gamma, C)$ and $M(\Gamma)$ and just write $T$ and $M$.

Teichmüller's metric $d_{T}$ is given by

$$
d_{T}([0],[\mu])=\inf \frac{1}{2} \log \frac{1+\|\mu\|_{\infty}}{1-\|\mu\|_{\infty}},
$$

where the infimum is over all $\mu$ in the equivalence class $[\mu]$. (Note that since the group $\Gamma$ and the closed set $C$ determine the total space $M(\Gamma)$ and the equivalence relation on it, the metric $d_{T}$ also depends on $\Gamma$ and $C$.) The distance between an arbitrary pair of points $\left[\mu_{0}\right]$ and $\left[\mu_{1}\right]$ is defined by declaring that right composition is an isometry; that is, $d_{T}\left(\left[\mu_{0}\right],\left[\mu_{1}\right]\right)$ is equal to the distance $d_{T}([0],[\nu])$ where $\nu$ is the Beltrami coefficient of $f_{0} \circ f_{1}^{-1}$ and where $f_{0}$ and $f_{1}$ are solutions to (1) for $\mu_{0}$ and $\mu_{1}$. If $f_{0}$ and $f_{1}$ are replaced by $L_{0} \circ f_{0}$ and $L_{1} \circ f_{1}$, then the expression in (3) remains unchanged, even before taking the infimum, so $d_{T}$ does not depend on the normalization at the boundary.

The Poincaré metric for $\Delta$ can be defined in a similar way. We view the unit disk $\Delta$ as a group of Möbius transformations preserving $\Delta$ factored by the rotations, $R(z)=e^{i \theta} z$. The distance between a Möbius transformation $L$ 
and the identity is

$$
d_{P}([\mathrm{id}],[L])=\frac{1}{2} \log \frac{1+\left|L^{-1}(0)\right|}{1-\left|L^{-1}(0)\right|}
$$

and the distance $d_{P}\left(\left[L_{0}\right],\left[L_{1}\right]\right)$ is defined to be $d_{P}\left([\mathrm{id}],\left[L_{1} \circ L_{0}^{-1}\right]\right)$.

The metrics $d_{P}$ and $d_{T}$ have infinitesimal forms and can be recovered as integrals of their infinitesimal forms. Proofs of these assertions can be found in [4]. Assume the distance from 0 to $t$ in the unit disk is given by $d_{P}(0, t)=|t|+O\left(|t|^{2}\right)$. If $d_{P}$ is to be invariant under precomposition by Möbius transformations, it follows that

$$
d_{P}(z, z+t)=|t| /\left(1-|z|^{2}\right)+O\left(|t|^{2}\right) .
$$

Thus $|d z| /\left(1-|z|^{2}\right)$ is a global expression in the unit disk for the infinitesimal form of $d_{P}$.

The infinitesimal form for Teichmüller's metric involves the space $A$ of integrable holomorphic quadratic differentials. For universal Teichmüller space (the case where $\Gamma=\{$ identity $\}$ and $C$ is the whole circumference of the unit disk), the Banach space $A$ consists of functions $\varphi(z)$ holomorphic in $\Delta$ with finite norm where $\|\varphi\|=\iint|\varphi(z)| d x d y$ with the integration over $\Delta$. In the case that $T=T(\Gamma, C)$, then, by definition, $A=A(\Gamma, C)$ is the Banach space of functions $\varphi(z)$ with the following properties:

(i) $\varphi$ is a quadratic differential form for $\Gamma$, that is, $\varphi(B(z)) B^{\prime}(z)^{2}=\varphi(z)$ for all $B$ in $\Gamma$.

(ii) $\|\varphi\|$ is finite, where $\|\varphi\|=\iint|\varphi(z)| d x d y$ and the domain of integration is any fundamental domain in $\Delta$ for $\Gamma$.

(iii) $\varphi(z) d z^{2}$ is real along the boundary of $\Delta$ minus $C$.

The infinitesimal form of Teichmüller's metric $[7,9]$ is

$$
d_{T}([0],[t \mu])=\sup \left|t \operatorname{Re} \iint \mu(z) \varphi(z) d x d y\right|+O\left(t^{2}\right)
$$

where the constant in $O\left(t^{2}\right)$ depends only on $\|\mu\|_{\infty}$ and the supremum is over $\varphi$ in the unit ball of $A=A(\Gamma, C)$. Since postcomposition by a quasi-conformal homeomorphism of $\Delta$ induces an isometry for $d_{T}$, in a manner analogous to the computation of (5), we find a global expression for the infinitesimal form of Teichmüller's metric [4]:

$$
d_{T}([\mu],[\mu+t \nu])=t \sup \left|\operatorname{Re} \iint \varphi(w)\left[\frac{\nu}{1-|\mu|^{2}} \cdot \frac{1}{\theta}\right] d u d v\right|+O\left(t^{2}\right)
$$

where the supremum is over all holomorphic quadratic differentials of norm 1 in $A\left(\Gamma^{\mu}, C^{\mu}\right), C^{\mu}=f^{\mu}(C), \Gamma^{\mu}=f^{\mu} \circ \Gamma \circ\left(f^{\mu}\right)^{-1}, \theta=\bar{p} / p, p=f_{z}^{\mu}$, and the integration is over a fundamental domain for $\Gamma^{\mu}$.

To prove formula (6) one uses two inequalities of Reich and Strebel [9], which are forms of Grötzsch's inequality applied to the horizontal trajectory structure of a quadratic differential. Let $K_{0}$ be the dilatation of an extremal mapping in with the same boundary values as $f=f^{\mu}$ and $\varphi$ any element of $A$ of norm 1. Then

$$
\frac{1}{K_{0}} \leq \iint \frac{|1-\mu(z) \varphi(z) /| \varphi(z) \|^{2}}{1-|\mu(z)|^{2}}|\varphi(z)| d x d y .
$$


This gives a lower bound for $K_{0}$. An upper bound comes from

$$
K_{0} \leq \sup \iint \frac{|1+\mu(z) \varphi(z) /| \varphi(z) \|^{2}}{1-|\mu(z)|^{2}}|\varphi(z)| d x d y
$$

where the supremum is over all $\varphi$ in $A$ of norm 1 . Now consider the curve [t $\mu]$ in Teichmüller space, let $K_{0}(t)$ be the dilatation of an extremal mapping in the class of $f^{t \mu}$, and let $k_{0}(t)=\left(K_{0}(t)-1\right) /\left(K_{0}(t)+1\right)$. Notice that the derivative with respect to $t$ at $t=0$ of $\frac{1}{2} \log K_{0}(t)$ is equal to the derivative at $t=0$ of $k_{0}(t)$, provided these derivatives exist. But applying (I) to $t \mu$ and expanding out the absolute value in the numerator of the integrand yields

$$
k_{0}(t) \geq\left|t \operatorname{Re} \iint \mu \varphi d x d y\right|+O\left(t^{2}\right)
$$

for all $\varphi$ of norm 1 and (II) yields

$$
k_{0}(t) \leq \sup \left|t \operatorname{Re} \iint \mu \varphi d x d y\right|+O\left(t^{2}\right)
$$

where the supremum is over all $\varphi$ of norm 1 . This shows that $k_{0}(t)$ is differentiable at $t=0$ and verifies formula (6). This proof of the formula for the infinitesimal form of Teichmüller's metric is given by Reich and Strebel in [9]. The first verification of the formula for the infinite-dimensional cases was given by O'Byrne in [7].

The Teichmüller metric $\bar{d}$ on $Q S \bmod S$ is defined by

$$
\bar{d}(S f, S g)=\frac{1}{2} \text { inf } \log K\left(s_{1} \circ f \circ g^{-1} \circ s_{2}^{-1}\right)
$$

where the infimum is over all $s_{1}$ and $s_{2}$ in the group $S$ of symmetric homeomorphisms of a circle. In [5] it is shown that this metric is given by boundary dilatation. If $f$ is a quasi-symmetric homeomorphism, its boundary dilatation $B D(f)$ is obtained by looking at the smallest maximal dilatation of quasiconformal extensions of $f$ to a neighborhood $U$ of the boundary and taking the limit of these dilatations as $U$ shrinks to the boundary. Then Lemma 5.1 of [5] says that

$$
\bar{d}(S f, S g)=\frac{1}{2} \log B D\left(f \circ g^{-1}\right) .
$$

A sequence $\varphi_{n}$ in $A$ is called degenerating if the norm of each $\varphi_{n}$ is equal to 1 and if $\varphi_{n}$ converges to zero uniformly on compact subsets of the disk. We denote a degenerating sequence by $\left\{\varphi_{n}\right\}$. Then there are inequalities $\left(I^{\prime}\right)$ and $\left(\mathrm{II}^{\prime}\right)$ analogous to (I) and (II):

$$
\frac{1}{B D(f)} \leq \inf _{\left\{\varphi_{n}\right\}} \liminf _{n} \iint \frac{\left|1-\mu(z) \varphi_{n}(z) /\right| \varphi_{n}(z) \|^{2}}{1-|\mu(z)|^{2}}\left|\varphi_{n}(z)\right| d x d y
$$

and

$$
B D(f) \leq \sup _{\left\{\varphi_{n}\right\}} \lim _{n} \sup \iint \frac{\left|1+\mu(z) \varphi_{n}(z) /\right| \varphi_{n}(z) \|^{2}}{1-|\mu(z)|^{2}}|\varphi(z)| d x d y .
$$

The proof of $\left(I^{\prime}\right)$ follows immediately from the main inequality of Reich and Strebel [9]. (II') is an existence theorem; it asserts the existence of a degenerating sequence satisfying the inequality.

To prove $\left(\mathrm{II}^{\prime}\right)$ we appeal to a theorem of Fehlmann relating local.dilatation to boundary dilatation. Consider a neighborhood $U$ of a point $\zeta$ on the boundary 
of the unit disk. Let $K_{\zeta}(f, U)$ be the infimum of the dilatations in $U$ of any mappings $g$, which agree with $f$ on the part of the boundary in common with the boundary of $U$. Then the local dilatation $B D_{\zeta}(f)$ of $f$ at $\zeta$ is the infimum over all neighborhoods $U$ of $\zeta$ of $K_{\zeta}(f, U)$. Fehlmann's theorem says that in all cases $B D(f)=\sup B D_{\zeta}(f)$ where the supremum is over all $\zeta$ in the boundary of the disk. We claim that $\left(\mathrm{II}^{\prime}\right)$ follows from the main inequality in [9] and this result. Since $B D_{\zeta}(f)$ is an upper semicontinuous function of $\zeta$, we can find a point $\zeta$ where $B D_{\zeta}(f)=B D(f)$. Then for any neighborhood $U$ of $\zeta$ we can choose a large finite set in $U \cap \partial \Delta$ such that the dilatation of an extremal mapping, which agrees with $f$ on this finite set, is as near to $B D_{\zeta}(f)$ as we like. This extremal mapping will have a Teichmüller Beltrami coefficient of the form $k|\varphi| / \varphi$ where $\|\varphi\|=1$ and all of the poles $\varphi$ are in the given finite set. This implies that most of the mass of the measure $|\varphi| d x d y$ is near to $\zeta$ because of the following lemma.

Lemma. Suppose $\varphi$ is a rational function with at most simple poles and $|\varphi(z)|=$ $O\left(|z|^{-4}\right)$ as $z \mapsto \infty$. Suppose also that all of the poles of $\varphi$ lie inside the disk $|z|<\varepsilon^{2}$ and that $\iint_{\mathbf{C}}|\varphi| d x d y=1$. Then

$$
\iint_{|z|<\varepsilon}|\varphi| d x d y \geq 1-2 \varepsilon
$$

Proof. First one verifies that for any function $\psi$ holomorphic in the unit disk

$$
\iint_{|\zeta|<c}|\psi| d \xi d \eta \leq \frac{c^{2}}{(1-c)^{2}} \iint_{|\zeta|<1}|\psi| d \xi d \eta
$$

Obviously, the left-hand side is less than or equal to $\pi c^{2}$ times the supremum of $\left|\psi\left(\zeta_{0}\right)\right|$ over $\left|\zeta_{0}\right|<c$. But if $\left|\zeta_{0}\right|<c$ then the disk of radius $1-c$ centered at $\zeta_{0}$ is contained in $|\zeta|<1$. Thus, by the mean value property for holomorphic functions,

$$
\left|\psi\left(\zeta_{0}\right)\right|<\frac{1}{\pi(1-c)^{2}} \iint_{|\zeta|<1}|\psi| d \xi d \eta
$$

and (8) follows.

Consider the change of coordinates $\zeta=\varepsilon \delta / z$ and let $\varphi(z)=\psi(\varepsilon \delta / z)(\varepsilon \delta)^{2} / z^{4}$. If all of the poles of $\varphi$ are inside the disk of radius $\varepsilon \delta$, all of the poles of $\psi$ are outside the unit disk and, since $|\varphi(z)|=O\left(|z|^{-4}\right), \psi(\zeta)$ is holomorphic at $\zeta=0$. We conclude that $\psi(\zeta)$ is holomorphic for $|\zeta|<1$.

Moreover,

$$
\iint_{D_{1}}|\varphi| d x d y=\iint_{D_{2}}|\psi| d \xi d \eta
$$

for any domain $D_{2}$, which is the image under the map $z \mapsto \zeta$ of the domain $D_{1}$. Hence,

$$
\iint_{|z|>\delta}|\varphi| d x d y=\iint_{|\zeta|<\varepsilon}|\psi| d \xi d \eta \leq \frac{\varepsilon^{2}}{(1-\varepsilon)^{2}} \iint_{|\zeta|<1}|\psi| d \xi d \eta \leq \frac{\varepsilon^{2}}{(1-\varepsilon)^{2}} .
$$

The last inequality holds because the hypothesis that $\iint_{\mathbf{C}}|\varphi| d x d y=1$ implies that $\iint_{\mathbf{C}}|\psi| d \xi d \eta=1$. Subtracting one from both sides of this inequality and changing signs yields $\iint_{|z|<\delta}|\varphi| d x d y \geq 1-2 \varepsilon$, and putting $\varepsilon=\delta$ yields the lemma. 
Now take a sequence of neighborhoods $U_{n}$, which shrink to a point $\zeta$ and take the Teichmüller Beltrami differentials $k_{n}\left|\varphi_{n}\right| / \varphi_{n}$, which realize the extremal mapping coinciding with $f$ at a finite number of points in $U_{n}$. We may choose these points so that the numbers

$$
K_{n}=\left(1+k_{n}\right) /\left(1-k_{n}\right)
$$

more and more nearly realize the boundary dilatation at $\zeta$ as $n \rightarrow \infty$. The sequence $\varphi_{n}$ has norm 1 , and all of the poles of $\varphi_{n}$ are contained in $U_{n}$. The fact that $\infty$ is not a pole of $\varphi_{n}$, considered as a quadratic differential, means that $|\varphi(z)| \leq O\left(|z|^{-4}\right)$ as $z \rightarrow \infty$. Therefore, $\varphi_{n}$ is a degenerating sequence because, from the lemma, larger and larger proportions of the mass for the measure $\left|\varphi_{n}\right| d x d y$ approach the point $\zeta$.

This is how one can find a degenerating sequence $\left\{\varphi_{n}\right\}$, which realizes the inequality in $\left(\mathbf{I I}^{\prime}\right)$. Given the inequalities $\left(\mathbf{I}^{\prime}\right)$ and $\left(\mathbf{I I}^{\prime}\right)$, formulas for the metric $\bar{d}$ on $Q S$ mod $S$ analogous to (6) and (7) for the metric $d$ on $Q S \bmod \operatorname{PSL}(2, \mathbf{R})$ are immediate consequences. Moreover, one verifies in the same way as in $[4$, p. 137$]$ that the metric $\bar{d}$ is the integral of its infinitesimal form. We state these results as theorems.

Theorem 1.1. The infinitesimal form of Teichmüller's metric $\bar{d}$ on $Q S \bmod S$ at the origin is

$$
\bar{d}\left(S, S f^{t \mu}\right)=\sup _{\left\{\varphi_{n}\right\}} \lim _{n} \sup \left|t \operatorname{Re} \iint \mu(z) \varphi(z) d x d y\right|+O\left(t^{2}\right)
$$

where the supremum is taken over all degenerating sequences $\varphi_{n}$ of norm 1 .

Theorem 1.2. The Teichmüller metric $\bar{d}$ on $Q S \bmod S$ is the integral of its infinitesimal form.

\section{Extremal Beltrami COEFficients AND Teichmüller CONTRACTION}

In the statement of the principle of Teichmüller contraction, we use $d_{P}$ to denote the Poincare metric on the unit disk and $d_{T}$ to denote the Teichmüller metric if $T$ is either universal Teichmüller space, the Teichmüller space of a Fuchsian group, or $Q S \bmod S$.

Principle of Teichmüller contraction. Assume $\|\mu\|_{\infty}=1$ and $0<k_{1}<k_{2}<1$ and $d_{T}\left(0, k_{1} \mu\right) \leq \lambda_{1} d_{p}\left(0, k_{1}\right)$ where $\lambda_{1}<1$. Then there exists a $\lambda_{2}<1$ depending only on $k_{1}, k_{2}$, and $\lambda_{1}$ such that

$$
d_{T}(0, k \mu) \leq \lambda_{2} d_{P}(0, k)
$$

for all $\mu$ with $\|\mu\|_{\infty}=1$ and all $k$ with $0 \leq k \leq k_{2}$.

Roughly speaking, the principle says that if the mapping $t \mapsto[t \mu]$ as a mapping from the unit disk with the Poincare metric to Teichmüller space with Teichmüller's metric is strictly contracting at two given points, then it is strictly contracting on all pairs of points, which are a bounded distance away from the two given points. The same constant of contraction applies at all scales, no matter how small, and for all $\mu$ with $\|\mu\|_{\infty}=1$. 


\section{EXTREMALITY OF MAPPINGS AND FUNCTIONALS}

We need to introduce functionals depending on $\mu$, which are used by Reich and Strebel in [9]. Assume $\|\mu\|_{\infty}=1$ and $0<k<1$. Let

$$
I(k \mu)=\sup \left|\operatorname{Re} \iint \frac{k \mu \varphi}{1-|k \mu|^{2}} d x d y\right|, \quad H(k \mu)=\sup \left|\operatorname{Re} \iint k \mu \varphi d x d y\right|
$$

where both suprema are taken over all $\varphi$ of norm 1 in $A$. Let $k_{0}$ be the smallest value of $\|\nu\|_{\infty}$ such that $f^{\nu}$ is in the same Teichmüller class as $f^{k \mu}$.

Definition. (a) The inefficiency at $k$ is $k-k_{0}$.

(b) The $i$-value at $k$ is $k /\left(1-k^{2}\right)-I(k \mu)$.

(c) The $h$-value at $k$ is $k-H(k \mu)$.

Proposition 3.1. The $i$-value, the $h$-value, and the inefficiency at $k$ are all comparable quantities. More precisely, fix $0<k_{1}<1$. Then there exist constants $C_{1}, C_{2}, C_{3}$, and $C_{4}$, which are independent of $\mu$ such that

(i) $C_{2}$ inefficiency at $k \leq i$-value at $k \leq C_{1}$ inefficiency at $k$,

(ii) $C_{3} h$-value at $k \leq i$-value at $k \leq C_{4} h$-value at $k$

for all $k$ with $0<k<k_{1}$. Here, $C_{1}=\left(1-k_{1}\right)^{-2}, C_{2}=1 / 4, C_{3}=\left(1-k_{1}\right)^{2} / 2$, and $C_{4}=1$.

Proof of Proposition 3.1. We use the inequalities (I) and (II) to prove part (i) of the proposition. From (I), we get

$$
\frac{1}{K_{0}}-\frac{1}{K} \leq \iint\left\{\frac{|1-k \mu(z) \varphi(z) /| \varphi(z) \|^{2}}{1-|k \mu(z)|^{2}}-\frac{1}{K}\right\}|\varphi(z)| d x d y
$$

for every $\varphi$ of norm 1 . On expanding the numerator of the first term in curly brackets, simplifying and dividing both sides of this inequality by two, one obtains

$$
\frac{k-k_{0}}{\left(1+k_{0}\right)(1+k)} \leq \iint\left\{\frac{k}{1+k}-\frac{\operatorname{Re} k \mu \varphi /|\varphi|}{1-|k \mu|^{2}}+\frac{|k \mu|^{2}}{1-|k \mu|^{2}}\right\}|\varphi(z)| d x d y .
$$

Since $\|\mu\|_{\infty}=1$, we obtain

$$
\frac{1}{4}\left(k-k_{0}\right) \leq \frac{k}{1-k^{2}}-\operatorname{Re} \iint \frac{\operatorname{Re} k \mu \varphi}{1-|k \mu|^{2}} d x d y
$$

for all $\varphi$ of norm 1, which implies the left-hand side of (i) of the proposition with $C_{2}=1 / 4$.

To obtain the right-hand side, we start with inequality (II) and find

$$
\frac{1}{2}\left(K-K_{0}\right) \geq \frac{k}{1-k^{2}}-\sup \operatorname{Re} \iint \frac{\operatorname{Re} k \mu \varphi}{1-|k \mu|^{2}} d x d y
$$

where the supremum is over all $\varphi$ of norm 1 . This gives the right-hand part of (i) with $C_{1}=1 /\left(1-k_{1}\right)^{2}$.

To prove part (ii) we rewrite the $i$-value at $k$ and the $h$-value at $k$ as

$$
i(k)=\inf \iint\left[\frac{k}{1-k^{2}}-\frac{\operatorname{Re} k \mu \varphi}{1-|k \mu|^{2}}\right]|\varphi| d x d y
$$

and

$$
h(k)=\inf \iint\{k-\operatorname{Re} k \mu \varphi\}|\varphi| d x d y,
$$


where both infima are taken over all $\varphi$ of norm 1. In order to compare the square bracket term in (8) with the curly bracket term in (9), we use the following.

Lemma. Assume $0 \leq k \leq k_{1}<1$ and $|z|<1$ where $z=x+i y$. Then

$$
k-k x \leq \frac{k}{1-k^{2}}-\frac{k x}{1-k^{2}|z|^{2}} \leq \frac{2}{\left(1-k_{1}\right)^{2}}(k-k x) .
$$

Proof. For the case when $x$ is between 0 and 1, we apply calculus to the function $f(t)=t /\left(1-t^{2}\right)$ for $0 \leq t<1$. The other cases are trivial.

Part (ii) of the proposition follows from this lemma.

Proposition 3.1 applies to universal Teichmüller space and Teichmüller space of a Fuchsian group. A parallel proposition with parallel definitions and parallel proof applies to $Q S \bmod S$. Define functionals $\bar{I}$ and $\bar{H}$ by

$$
\bar{I}(k \mu)=\sup _{\left\{\varphi_{n}\right\}} \lim _{n} \sup \left|\operatorname{Re} \iint \frac{k \mu \varphi_{n}}{1-|k \mu|^{2}} d x d y\right|
$$

and

$$
\bar{H}(k \mu)=\sup _{\left\{\varphi_{n}\right\}} \lim _{n} \sup \left|\operatorname{Re} \iint k \mu \varphi_{n} d x d y\right|,
$$

where both suprema are taken over all degenerating sequences $\varphi_{n}$ of norm 1. Let $\bar{K}=\bar{K}\left(f^{k \mu}\right)=\inf K\left(f^{k \mu}, U\right)$ where the infimum is taken over all annular neighborhoods $U$ of the boundary of the unit disk and $K\left(f^{k \mu}, U\right)$ means the dilatation of $f^{k \mu}$ restricted to $U$. Let $\bar{k}=(\bar{K}-1) /(\bar{K}+1)$. Let $\bar{k}_{0}=\inf \bar{k}(g)$ where the infimum is over all quasi-conformal mappings $g$ with the same boundary values as $f^{k \mu}$.

Definitions. Assume $\mu$ is a Beltrami coefficient with $\|\mu\|_{\infty}=1$ and $0 \leq k<1$. Then for the Teichmüller space $Q S \bmod S$ :

(a) the inefficiency of $\mu$ at $k$ for $Q S \bmod S$ is $\bar{k}-\bar{k}_{0}$,

(b) the $i$-value of $\mu$ at $k$ for $Q S \bmod S$ is $\bar{k} /\left(1-(\bar{k})^{2}\right)-\bar{I}(k \mu)$,

(c) the $h$-value of $\mu$ at $k$ for $Q S \bmod S$ is $\bar{k}-\bar{H}(k \mu)$.

Proposition 3.2. All of the assertions of Proposition 3.1 hold in the setting of $Q S \bmod S$.

Corollary (The Hamilton-Reich-Strebel condition for extremality in $Q S$ mod $S$ ). For a Beltrami coefficient $\mu, \bar{k}=\bar{k}_{0}$ if and only if

$$
\sup _{\left\{\varphi_{n}\right\}} \lim _{n} \sup \left|\operatorname{Re} \iint \mu \varphi_{n} d x d y\right|=\bar{k}\left(f^{\mu}\right),
$$

where the supremum is over all degenerating sequences $\left\{\varphi_{n}\right\}$ with $\left\|\varphi_{n}\right\|=1$.

\section{Proof of the Teichmüller contraction}

Assume we have a value $k_{1}$ such that the Beltrami line associated with $\mu$ is inefficient at $k_{1}$ in the sense that $d_{T}\left(0, k_{1} \mu\right) \leq \lambda_{1} d_{P}\left(0, k_{1}\right)$. Let $k_{0}$ be the extremal value of $\|\nu\|_{\infty}$ where $\nu$ is equivalent to $k_{1} \mu$. Then

$$
\frac{1}{2} \log \frac{1+k_{0}}{1-k_{0}} \leq \frac{1}{2} \lambda_{1} \log \frac{1+k_{1}}{1-k_{1}}
$$


which means that the inefficiency at $k_{1}$ is a positive number $b$, which can be estimated in terms of $\lambda_{1}$ and $k_{1}$. From part (i) of Proposition 3.1, we get

$$
\frac{1}{4} b \leq \frac{k_{1}}{1-k_{1}^{2}}-I\left(k_{1} \mu\right)
$$

From part (ii) of the same proposition, it follows that $b_{1} \leq h\left(k_{1}\right)$ where $b_{1}=$ $\left(1-k_{1}^{2}\right) b / 4$. Thus, $h(t k-1) \geq t b_{1}$ for $t \geq 0$. Using the proposition again we find that $i(k) \geq k b_{2}$ for $0 \leq k \leq k_{2}$. Rewriting this result, it becomes

$$
I(k \mu) \leq \frac{k}{1-k^{2}}-k b_{2} \leq \frac{k}{1-k^{2}}-\frac{b_{2} k}{1-k^{2}} \cdot\left(1-k_{2}^{2}\right) \leq\left(1-b_{3}\right) \frac{k}{1-k^{2}}
$$

where $b_{3}=b_{2}\left(1-k_{2}^{2}\right)$ for $0 \leq k \leq k_{2}$.

Once again, using part (i) of the proposition, we get a bound on the inefficiency at $k$ for all values with $0 \leq k \leq k_{2}$. Alternatively, we could apply (10) to the Beltrami coefficient of the inverse mapping to $f^{k \mu}$, integrate the infinitesimal form of Teichmüller's metric (7), and obtain

$$
d_{T}(0, k \mu) \leq\left(1-b_{3}\right) d_{P}(0, k)
$$

for $0 \leq k \leq k_{2}$. The proof of the Teichmüller contraction for $Q S \bmod S$ follows in the same way.

Remark. We can view Proposition 3.1 as an $\varepsilon \varepsilon^{\prime}$-version of the Hamilton-ReichStrebel necessary and sufficient condition for extremality. There is a sufficient condition due to Reich [8] for a Beltrami coefficient to be uniquely extremal; there may be an $\varepsilon \varepsilon^{\prime}$-version of this condition as well. Although it is an oxymoron, there is a notion of "nearly unique". We can say an extremal quasiconformal mapping $f_{1}$ is $\varepsilon$-nearly unique if for any other extremal mapping $f_{2}$ with the same boundary values, the distance in the Poincare metric from $f_{1}(p)$ to $f_{2}(p)$ is less than $\varepsilon$ for all $p$ in the disk. Reich's functional can also be put into an $\varepsilon^{\prime}$-form. An obvious guess would be that $\varepsilon$ approaches zero if $\varepsilon^{\prime}$ does.

\section{ACKNOWLEDGMENT}

I wish to acknowledge many helpful discussions with Dennis Sullivan, who formulated the principle of Teichmüller contraction for structure preserving mappings between Teichmüller spaces. He applies this principle to renormalization mappings and to Riemann surface laminations; an exposition appears in the appendix of [11].

\section{REFERENCES}

1. L. V. Ahlfors and L. Bers, Riemann's mapping theorem for variable metrics, Ann. of Math. (2) 72 (1960), 385-404.

2. R. Fehlmann, Extremal quasiconformal mappings with free boundary components in domains of arbitrary connectivity, Math. Z. 184 (1983), 109-126.

3. F. G. Gardiner, Approximation of infinite dimensional Teichmüller spaces, Trans. Amer. Math. Soc. 282 (1984), 367-383.

4. __ Teichmüller theory and quadratic differentials, Wiley-Interscience, New York, 1987.

5. F. P. Gardiner and D. P. Sullivan, Symmetric and quasisymmetric structures on a closed curve, Amer. J. Math. 114 (1992), 683-736. 
6. O. Lehto and K. I. Virtanen, Quasiconformal mappings, Springer-Verlag, Berlin and New York, 1965.

7. B. O'Byrne, On Finsler geometry and applications to Teichmüller spaces, Ann. of Math. Stud., vol. 66, Princeton Univ. Press, Princeton, NJ, 1971, pp. 317-328.

8. E. Reich, On criteria for unique extremality of Teichmüller mappings, Ann. Acad. Sci. Fenn. AI Math. 6 (1981), 289-302.

9. E. Reich and K. Strebel, Extremal quasiconformal mappings with given boundary values, Contributions to Analysis, A Collection of Papers Dedicated to Lipman Bers, Academic Press, New York, 1974, pp. 373-391.

10. H. L. Royden, Automorphisms and isometries of Teichmüller space, Ann. of Math. Stud., vol. 66, Princeton Univ. Press, Princeton, NJ, 1971, pp. 369-383.

11. D. P. Sullivan, Bounds, quadratic differentials, and renormalization conjectures, Mathematics into the 21st Century, Amer. Math. Soc. Centennial Publication, vol. 2, Amer. Math. Soc., Providence, RI, 1991.

Department of Mathematics, City University of New York, Brooklyn College, BROOKLYN, NEW YORK 11210

E-mail address: FPGBC@CUNYVM.BITNET 